\title{
Análise de trilha de caracteres forrageiros do capim-elefante (Pennisetum purpureum Schum.)
}

\author{
Path coefficient analysis of forage characteristics of elephantgrass \\ (Pennisetum purpureum schum.)
}

\author{
Rogério Figueiredo Daher ${ }^{1}$ Antônio Vander Pereira ${ }^{2}$ Messias Gonzaga Pereira \\ Francisco José da Silva Lédo ${ }^{2}$ Antônio Teixeira do Amaral Junior ${ }^{3}$ \\ Juan Manuel Anda Rocabado ${ }^{4}$ Cláudia Fortes Ferreira ${ }^{5}$ \\ Flávio Dessaune Tardin' 6 .
}

\section{RESUMO}

Os objetivos deste trabalho consistiram em obter estimativas de coeficientes de correlação fenotípicos, genotípicos e residuais, e avaliar os desdobramentos das correlações genotípicas em efeitos diretos e indiretos (análise de trilha) das variáveis altura média das plantas, diâmetro médio do colmo e número de perfilhos por metro linear (variáveis independentes explicativas) na produção de matéria seca (variável dependente principal) de clones de capim-elefante em dois cortes realizados nas condições edafoclimáticas do norte do Estado do Rio de Janeiro. Houve grande diferença nas estimativas obtidas nos dois cortes, entretanto o estudo permitiu concluir que a altura das plantas no corte exerce influência na produção de matéria seca principalmente nas situações de clones de alta capacidade de perfilhamento. As características número de perfilhos por metro linear e diâmetro de perfilhos foram capazes de explicar melhor o potencial de produção de matéria seca, atuando, respectivamente, de forma direta e inversamente proporcionais sobre a variável básica, alternando-se em função das condições ambientais ocorridas durante o crescimento.

Palavras-chave: correlação genotípica, melhoramento genético, clone, produção de matéria seca.

\section{ABSTRACT}

The main goals of this work was to obtain estimates of phenotype, genotype and residual correlation coefficients and display genotype correlations in direct and indirect effects (path analysis) of height, diameter of stem at the base and number of tillers per meter (explanatory independent variables) on dry matter production (basic dependent variable) of elephantgrass clones in two harvest periods at conditions of northern Rio de Janeiro State, Brazil. Great differences among estimates in two harvest periods were observed, however it could be concluded that height of plants at cutting influenced dry matter production mainly in conditions of high tillering capable clones. Number of tillers per meter and diameter of stem explained better dry matter production potential, acting, respectively, in an direct and inverse way, alternating according to environmental conditions during growth.

Key words: genetic correlation, genetic breeding, clone, dry matter production.

\section{INTRODUÇÃO}

O conhecimento dos relacionamentos existentes entre as variáveis empregadas para o melhoramento da espécie de interesse é de suma importância uma vez que se necessita obter ganhos não para uma única variável, mas para um conjunto delas. A correlação entre duas variáveis pode ser de natureza fenotípica, genotípica ou ambiental, sendo que somente as correlações genotípicas envolvem uma associação de natureza herdável, sendo de grande interesse para o melhoramento.

Entretanto, as correlações não são medidas de causa e efeito e a interpretação direta

\footnotetext{
${ }^{1}$ Engenheiro Agrônomo, Doutor, Técnico do Laboratório de Melhoramento Genético Vegetal (LMGV), Centro de Ciências e Tecnologias Agropecuárias (CCTA), Universidade Estadual do Norte Fluminense Darcy Ribeiro (UENF). Av. Alberto Lamego, 2000, Parque Califórnia, 28013-600, Campos dos Goytacazes, RJ. Tel.: 22-2726-1432. E-mail: rogdaher@uenf.br. Autor correspondente. ${ }^{2}$ Engenheiro Agrônomo, Doutor, Pesquisador da Embrapa Gado de Leite. Rua Eugênio do Nascimento, 610, 36038-330, Juiz de Fora, MG.

${ }^{3}$ Engenheiro Agrônomo, PhD, Professor Associado do LMGV, CCTA, UENF.

${ }^{4}$ Engenheiro Agrônomo, Doutor, Bolsista do Laboratório de Proteção de Plantas, LPP, CCTA, UENF.

${ }^{5}$ Engenheiro Agrônomo, Doutor, Pesquisador da Embrapa Mandioca e Fruticultura. R. Embrapa, s/n. CP 007, 44380-000, Cruz das Almas, BA.

${ }^{6}$ Engenheiro Agrônomo, Doutorando em Produção Vegetal, LMGV, CCTA, UENF.
} 
das suas magnitudes pode resultar em equívocos na estratégia de seleção, pois correlação alta entre dois caracteres pode ser resultados do efeito, sobre estes, de um terceiro caráter ou de um grupo de caracteres (DUNTEMAN, 1984), sendo pois necessário o emprego de metodologias específicas, como as correlações parciais, correlações canônicas e análise de trilha (FALCONER, 1987; CRUZ \& REGAZZI, 1997).

A análise de trilha, desenvolvida por WRIGHT (1921 e 1923) consiste no estudo dos efeitos diretos e indiretos de caracteres independentes explicativos sobre uma variável dependente principal básica, cujas estimativas são obtidas por meio de equações de regressão, em que as variáveis são previamente padronizadas.

Esta técnica têm sido amplamente utilizada no melhoramento de diversas culturas, citando FURTADO et al. (2002) em feijoeiro, CARVALHO et al. (1999) em pimentão e CORREIA et al. (1996) em eucalipto, dentre outros. Entretanto, ainda são escassos trabalhos desta natureza envolvendo plantas forrageiras e, em especial, o capim-elefante.

DIZ et al. (1994) examinaram, sob vários tratamentos de desfolha, em dois anos consecutivos, os efeitos diretos e indiretos do número de panículas por planta, da produção de semente por panícula e do peso de 100 sementes na produção de sementes por planta de híbridos de capim-elefante com milheto. Concluíram que híbridos de Pennisetum devem ser selecionados para maior produção de sementes por planta, uma vez que esta característica é relativamente de fácil mensuração e está altamente correlacionada com a produção de sementes por planta.

O capim-elefante é também conhecido pelos nomes de capim-napier, cameroon e capim-cana, que, na verdade, são designações de cultivares. Foi introduzida no Brasil por volta de 1920, por meio de mudas provenientes de Cuba (CARVALHO, 1985), e é, atualmente uma das forrageiras mais difundidas em todo o país.

Os objetivos deste trabalho consistiram em obter estimativas de coeficientes de correlação fenotípicos, genotípicos e residuais e avaliar os desdobramentos das correlações genotípicas em efeitos diretos e indiretos das variáveis altura média das plantas, diâmetro médio do colmo e número de perfilhos por metro linear (variáveis independentes explicativas) na produção de matéria seca (variável dependente principal) de clones de capim-elefante em 2 cortes realizados nas condições edafoclimáticas do norte do Estado do Rio de Janeiro.

\section{MATERIAL E MÉTODOS}

O experimento foi conduzido nas dependências do Colégio Estadual Agrícola Antônio Sarlo, em Campos dos Goytacazes, Norte do Rio de Janeiro, RJ, num solo de terraço, classificado como Latossolo Amarelo, distrófico, textura arenosa, no período de fevereiro de 1995 a dezembro de 1997, totalizando 12 cortes. As avaliações foram realizadas no quarto (23/04/1996) e no sétimo (18/12/1996) cortes, com respectivos períodos de crescimento de 65 e 56 dias, selecionados ao acaso dentre os cortes realizados. Foram utilizados dezessete clones (híbridos intraespecíficos) de capim-elefante (Pennisetum purpureum Schum.) provenientes do Programa de Melhoramento da Embrapa Gado de Leite, Juiz de Fora, MG. O delineamento experimental utilizado foi blocos completos casualizados, com quatro repetições. A parcela foi composta por 4 linhas de quatro metros, espaçadas de um metro, sendo considerado como área útil os dois metros centrais das duas linhas do interior da parcela, totalizando quatro metro quadrados.

Procedeu-se à calagem na dose de 2 t.ha ${ }^{-1}$ de calcário dolomítico. O plantio foi realizado em 17/ $02 / 95$, por meio de plantas inteiras, dispostas pé com ponta, em fileiras duplas, em sulcos de $10 \mathrm{~cm}$ de profundidade, acompanhado da seguinte adubação: 100kg.ha' ${ }^{-1}$ de $\mathrm{P}_{2} \mathrm{O}_{5}, 30 \mathrm{~kg} \cdot \mathrm{ha}^{-1}$ de $\mathrm{K}_{2} \mathrm{O}$ e $15 \mathrm{~kg}$.ha ${ }^{-1}$ de N, incorporada no fundo do sulco. Após 50 dias do plantio, complementou-se a adubação com cobertura de $30 \mathrm{~kg} \cdot \mathrm{ha}^{-1}$ de $\mathrm{K}_{2} \mathrm{O}$ e $25 \mathrm{~kg} \cdot \mathrm{ha}^{-1}$ de $\mathrm{N}$ e, em 22/06/95, realizou-se o corte de uniformização. Após cada corte, foi efetuada adubação em cobertura com $60 \mathrm{~kg} \cdot \mathrm{ha}^{-1} \mathrm{de}$ $\mathrm{K}_{2} \mathrm{O}$ e $50 \mathrm{~kg}$.ha ${ }^{-1}$ de $\mathrm{N}$ e uma adubação fosfatada no início da época das águas (100kg.ha-1 $\left.\mathrm{de}_{2} \mathrm{O}_{5}\right)$.

As características avaliadas foram produção de matéria seca (MS), em kg.ha-1 ${ }^{-1}$ corte (PMS) (variável dependente principal ou básica) e as variáveis independentes explicativas altura média das plantas, expressa em m (ALT); diâmetro médio do colmo, expresso em cm, tomado a uma altura de $10 \mathrm{~cm}$ do solo, por meio de um paquímetro (DP); e número de perfilhos por metro linear (NP).

Os estimadores dos coeficientes de correlações fenotípicas, genotípicas e de ambiente foram obtidos pelas expressões:

a) Correlações fenotípicas

b) Correlações genotípicas

$$
\mathrm{r}_{\mathrm{F}}=\frac{\mathrm{PMG}_{\mathrm{XY}}}{\sqrt{\mathrm{QMG}_{\mathrm{X}} \cdot \mathrm{QMG}_{\mathrm{Y}}}}
$$

$$
\mathrm{r}_{\mathrm{G}}=\frac{\left(\mathrm{PMG}_{\mathrm{XY}}-\mathrm{PMR}_{\mathrm{XY}}\right) / \mathrm{r}}{\sqrt{\hat{\phi}_{\mathrm{g}(\mathrm{X})} \hat{\phi}_{\mathrm{g}(\mathrm{Y})}}}=\frac{\hat{\phi}_{\mathrm{g}(\mathrm{XY})}}{\sqrt{\hat{\phi}_{\mathrm{g}(\mathrm{X})} \hat{\phi}_{\mathrm{g}(\mathrm{Y})}}}
$$

Ciência Rural, v.34, n.5, set-out, 2004. 
c) Correlações de ambiente

$$
r_{\mathrm{A}}=\frac{\mathrm{PMR}_{\mathrm{XY}}}{\sqrt{\mathrm{QMR}_{\mathrm{X}} \mathrm{QMR}_{\mathrm{Y}}}}
$$

em que:

$\mathrm{PMG}_{\mathrm{XY}}$ : produto médio entre os genótipos para os caracteres X e Y;

$\mathrm{PMR}_{\mathrm{XY}}$ : produto médio entre os resíduos para os caracteres X e Y;

$\mathrm{QMG}_{\mathrm{X}}$ : quadrado médio entre os genótipos para o caráter $\mathrm{X}$;

$\mathrm{QMG}_{\mathrm{Y}}$ : quadrado médio entre os genótipos para o caráter Y;

$\mathrm{QMR}_{\mathrm{X}}$ : quadrado médio entre os resíduos para o caráter X;

$\mathrm{QMR}_{\mathrm{Y}}$ : quadrado médio entre os resíduos para o caráter Y;

$\hat{\varphi}_{\mathrm{g}_{(\mathrm{XY})}}$ : estimador da covariância genotípica;

$$
\begin{gathered}
\hat{\phi}_{\mathrm{g}_{(\mathrm{X})}}=\frac{\mathrm{QMG}_{\mathrm{X}}-\mathrm{QMR} \mathrm{R}_{\mathrm{X}}}{\mathrm{r}} \\
\hat{\phi}_{\mathrm{g}_{(\mathrm{Y})}}=\frac{\mathrm{QMG}_{\mathrm{Y}}-\mathrm{QMR}}{\mathrm{r}}
\end{gathered}
$$

em que $\hat{\phi}_{g(x)}$ e $\hat{\phi}_{\mathrm{g}_{(\mathrm{Y})}}$ estimadores dos componentes quadráticos associados às variabilidades genotípicas para os caracteres X e Y, respectivamente.

Realizou-se diagnóstico de multicolinearidade envolvendo as quatro variáveis resultando em fraca colinearidade. Os resultados da análise de trilha foram interpretados conforme indicado por SINGH \& CHAUDARY (1979), citado por VENCOVSKY \& BARRIGA (1992), em que, basicamente, i) coeficientes de correlação e efeitos diretos (coeficientes de trilha) altos indicam que estas variáveis independentes explicam grande parte da variação da variável básica e que ii) coeficientes de correlação positivos (ou negativos), mas o efeito direto é de sinal diferente ou negligenciável, variáveis com maiores efeitos indiretos devem ser consideradas simultaneamente para explicarem a variação da variável básica. Todas as análises estatísticas foram realizadas com o uso do programa computacional genes (CRUZ, 1997).

\section{RESULTADOS E DISCUSSÃO}

As estimativas das correlações genotípicas superaram as fenotípicas e ambientais para todos os pares de combinações nos dois cortes avaliados, exceto para o par envolvendo PMS e NP no corte "7", em que a estimativa da correlação ambiental $(0,5118)$ superou tanto a genotípica $(-0,1407)$ quanto a fenotípica $(0,1425)$ (Tabela 1). Nesta combinação (PMS e NP no corte “7”), a correlação genotípica não se apresentou significativamente diferente de zero, pelo teste $\mathrm{t}(\mathrm{P}>0,05)$, juntamente com o par ALT e DP, também no corte "7" ( $\left.r_{G}=-0,2177\right)$ (Tabela 1$)$.

Verificaram-se diferenças em sinal envolvendo as correlações genotípicas e as de ambiente, mostrando que as causas de variação genética e de ambiente podem afetar os caracteres por meio de diferentes mecanismos fisiológicos, como o florescimento, por exemplo (FALCONER, 1987).

Inconsistências de estimativas de correlações genotípicas (e de significâncias do teste) foram observadas nas duas épocas (Tabela 1). PMS e NP exibiram correlação positiva $(\mathrm{P}<0,01)$ no corte $4 \mathrm{e}$ ausência de correlação ( $\mathrm{P}>0,05)$ no corte 7 , assim como ALT e NP apresentaram correlação positiva $(\mathrm{P}<0,01)$ no corte 4 e negativa $(\mathrm{P}<0,01)$ no corte 7 . DAHER et al. (2003), avaliando a estabilidade da produção forrageira (variável PMS) para os mesmos dezessete clones deste trabalho, relataram a ocorrência de interação clone $\mathrm{x}$ corte $(\mathrm{P}<0,05)$, indicando que períodos de crescimento diferentes podem provocar respostas diferentes no comportamento das características avaliadas.

As estimativas dos efeitos diretos e indiretos das variáveis independentes explicativas ALT, DP e NP sobre a produção de matéria seca, nos dois cortes avaliados, encontram-se na tabela 2. A altura das plantas, apesar dos elevados valores de correlação genotípica com a produção de matéria seca nos dois cortes avaliados $\left(\mathrm{r}_{\mathrm{G}}=0,6868\right.$ e 0,7489 , respectivamente para os cortes “4" e "7"), apresentou baixos valores de efeitos diretos $(0,3433$ e 0,2831$)$, sendo estes, inclusive, inferiores aos respectivos valores de efeito da variável residual: 0,3542 e 0,3241, o que reduz sua importância em relação às variáveis DPe NP. Verifica-se que o efeito indireto de ALT via NP no corte "4" $(0,5853)$ foi mais importante que o próprio efeito direto sobre a produção de matéria seca. A alta correlação genotípica entre ALT e NP $(0,4912)$ (Tabela 1$)$ é corroborada pela interação verificada por meio do efeito indireto de ALT via NP sobre a variável básica PMS (Tabela 2).

Trabalhos desenvolvidos com cana de açúcar (JAMES, 1971; MILLER \& JAMES, 1974; KANG et al., 1985) também indicaram, pela análise de trilha, com base em correlações genotípicas, que a característica altura das plantas apresentou-se menos importante em relação a diâmetro de caule e número de plantas como componente da variável básica produção de cana.

As variáveis DP e NP alternaram-se como mais importantes nos cortes avaliados. No corte "4", a 
Tabela 1 - Estimativas dos coeficientes de correlação fenotípica $\left(\mathbf{r}_{\mathrm{F}}\right)$, genotípica $\left(\mathbf{r}_{\mathrm{G}}\right.$ ) e ambiental $\left(\mathbf{r}_{\mathrm{A}}\right)$ entre 4 caracteres avaliados em 2 cortes em 17 genótipos de capim-elefante em Campos dos Goytacazes, RJ.

\begin{tabular}{|c|c|c|c|c|c|c|c|}
\hline & \multicolumn{2}{|c|}{$\mathrm{ALT}^{1 /}$} & \multicolumn{2}{|c|}{$\mathrm{DP}^{2 /}$} & \multicolumn{3}{|c|}{$\mathrm{NP}^{3 /}$} \\
\hline & $\mathrm{r}$ & 4 & 7 & 4 & 7 & 4 & 7 \\
\hline \multirow{3}{*}{$\mathrm{PMS}^{4 /}$} & $\mathrm{F}$ & 0,5032 & 0,5857 & $-0,1422$ & $-0,0776$ & 0,5862 & 0,1425 \\
\hline & G & $0,6868 * *$ & $0,7489 * *$ & $-0,3405^{* *}$ & $-0,5713 * *$ & $0,7493 * *$ & $-0,1407 \mathrm{~ns}$ \\
\hline & A & 0,3530 & 0,5310 & 0,1816 & 0,1659 & 0,4441 & 0,5118 \\
\hline \multirow{3}{*}{ ALT } & $\mathrm{F}$ & & & $-0,2634$ & $-0,0568$ & 0,3914 & $-0,2505$ \\
\hline & G & & & $-0,3240 * *$ & $-0,2177 \mathrm{~ns}$ & $0,4912 * *$ & $-0,3320 * *$ \\
\hline & A & & & 0,1146 & 0,1435 & 0,1344 & 0,0195 \\
\hline \multirow{3}{*}{ DP } & $\mathrm{F}$ & & & & & $-0,5797$ & $-0,3304$ \\
\hline & G & & & & & $-0,8186^{* *}$ & $-0,6824 * *$ \\
\hline & A & & & & & 0,0416 & $-0,0076$ \\
\hline
\end{tabular}

** - Significativamente diferente de zero $(\mathrm{P}<0,01)$; ns - não significativo $(\mathrm{P}>0,05)$ pelo teste $\mathrm{t}(\mathrm{GL}=66)$, aplicado somente às correlações genotípicas.

${ }^{1 /}$ ALT - Altura média das plantas; ${ }^{2 /} \mathrm{DP}$ - Diâmetro médio do colmo; ${ }^{3 /} \mathrm{NP}$ - Número de perfilhos por metro linear; ${ }^{4 /}$ PMS - Produção de matéria seca.

variável NP teve melhor desempenho, pela alta correlação genotípica verificada $(0,7493)$ aliado ao alto valor de efeito direto $(1,1915)$. Neste corte, a produção de matéria seca dos clones pode ser melhor explicada

Tabela 2 - Análise de trilha: desdobramento das correlações genotípicas em componentes de efeito direto e indiretos envolvendo a variável dependente principal PMS (produção de matéria seca, em kg.ha ${ }^{-1}$ ) e as variáveis independentes explicativas ALT, DP e NP avaliados em 2 cortes em 17 genótipos de capimelefante em Campos dos Goytacazes, RJ.

\begin{tabular}{lcc}
\hline Variável & \multicolumn{2}{c}{ Número do corte } \\
\hline ALT $^{1 /}$ & 4 & 7 \\
Efeito direto sobre PMS & 0,3433 & 0,2831 \\
Efeito indireto via DP & $-0,2418$ & 0,2206 \\
via NP & 0,5853 & 0,2451 \\
TOTAL & 0,6868 & 0,7489 \\
DP $^{2 /}$ & & \\
Efeito direto sobre PMS & 0,7462 & $-1,0136$ \\
Efeito indireto via ALT & $-0,1112$ & $-0,0616$ \\
via NP & $-0,9754$ & 0,5039 \\
TOTAL & $-0,3405$ & $-0,5713$ \\
NP & & \\
Efeito direto sobre PMS & 1,1915 & $-0,7384$ \\
Efeito indireto via ALT & 0,1686 & $-0,0940$ \\
via DP & $-0,6108$ & 0,6916 \\
TOTAL & 0,7493 & $-0,1407$ \\
Coeficiente de determinação $\left(\mathrm{R}^{2}\right)$ & 0,8745 & 0,8949 \\
\hline Efeito da variável residual & 0,3542 & 0,3241 \\
\hline
\end{tabular}

${ }^{1 /} \mathrm{ALT}$ - Altura média das plantas; ${ }^{2 /} \mathrm{DP}$ - Diâmetro médio do colmo; ${ }^{3 /} \mathrm{NP}$ - Número de perfilhos por metro linear; ${ }^{4 /} \mathrm{PMS}$ Produção de matéria seca. pela presença de elevado número de perfilhos por metro linear. Já no corte “7”, a variável DP foi capaz de explicar melhor PMS, no entanto, em sentido inverso, ou seja, os clones com reduzidos valores de diâmetro de perfilhos induziram a produções de matéria seca mais elevadas. Observou-se moderada correlação genotípica negativa ( $0,5713)$ aliado ao alto valor de efeito direto (-1,0136).

$\mathrm{O}$ coeficiente de determinação do modelo da análise de trilha $\left(\mathrm{R}^{2}\right)$ apresentou-se elevado em ambos os cortes, com valores de 0,8745 e 0,8949, indicando que as variáveis independentes explicativas ALT, DP e NP explicaram grande parte da variação da variável básica PMS.

\section{CONCLUSÕES}

Houve grande diferença nas estimativas obtidas nos dois cortes, entretanto o estudo permitiu concluir que a altura das plantas no corte exerce influência na produção de matéria seca principalmente nas situações de clones de alta capacidade de perfilhamento. As características número de perfilhos por metro linear e diâmetro de perfilhos a $10 \mathrm{~cm}$ do solo foram capazes de explicar melhor o potencial de produção de matéria seca, atuando, respectivamente, de forma direta e inversamente proporcionais sobre a variável básica, alternando-se em função das condições ambientais ocorridas durante o crescimento.

\section{REFERÊNCIAS BIBLIOGRÁFICAS}

CARVALHO, L.A. Pennisetum purpureum Schumacher: Revisão. Coronel Pacheco : EMBRAPA-CNPGL, 1985. 86p. (EMBRAPA - CNPGL, Boletim de Pesquisa, 10). 
CARVALHO, C.G.P. et al. Análise de trilha sob multicolinearidade em pimentão. Pesquisa Agropecuária Brasileira, Brasília, v.34, n.4, p.603-613, 1999.

CORREIA, J.R. et al. Análise de trilha ("path analysis") no estudo do relacionamento entre características físicas e químicas do solo e a produtividade do eucalipto. Revista Árvore, Viçosa, v.20, n.2, p.161-169, 1996.

CRUZ, C.D.; REGAZZI, A.J. Modelos biométricos aplicados ao melhoramento genético. Viçosa : UFV, 1997. 390 p.

CRUZ, C.D. Programa GENES: aplicativo computacional em genética e estatística. Viçosa : UFV, 1997. 442p.

DAHER, R.F et al. Estabilidade da produção forrageira de clones de capim-elefante (Pennisetum purpureum Schum.). Ciência e Agrotecnologia, Lavras, v.27, n.4, p.788-797, 2003.

DIZ, D.A.; WOFFORD, D.S.; SCHANK, S.C. Correlation and path-coefficient analysis of seed-yield component in pearl millet $x$ elephantgrass hybrids. Theoretical and Applied Genetics, Berlin, v.89, p.112-115, 1994.

DUNTEMAN, G.H. Introduction to multivariate analysis. Beverly Hills : Sage Publications, 1984. $237 \mathrm{p}$.
FALCONER, D.S. Introdução à genética quantitativa. Viçosa, MG : UFV, 1987. 279p.

FURTADO, M.R. et al. Análise de trilha do rendimento do feijoeiro e seus componentes primários em monocultivo e em consórcio com a cultura do milho. Ciência Rural, Santa Maria, v.32, n.2, p.217-220, 2002.

JAMES, N.I. Yield components in random and selected sugarcane populations. Crop Science, v.11, p.906-908, 1971.

KANG, M.S.; MILLER, J.D.; TAI, P.Y.P. Genetic and phenotypic path analysis and heritability in sugarcane. Crop Science, v.25, p.643-647, 1985.

MILLER, J.D.; JAMES, N.I. The influence of stalk density on cane yield. p. 177-184. In: INTERNATIONAL SOCIETY OF SUGAR CANE TECHOLOGY CONGRESS, 1974. South Africa. Proceedings... Durban, South Africa : Hayne and Gibbon Limited, 1974.

VENCOVSKY, R.; BARRIGA, P. Genética biométrica no fitomelhoramento. Ribeirão Preto, SP : Revista Brasileira de Genética, 1992. 496p

WRIGHT, S. Correlation and causation. Journal of Agricultural Research, Washington, v.20, n.7, p.557-585, 1921.

WRIGHT, S. Theory of path coefficients. Genetics, New York, v.8, p.239-285, 1923 